International Journal of Advanced Biological and Biomedical Research Available online at http:www.ijabbr.com

Volume 7, Issue 3 (2019) pp. 263-273

Review Article

\title{
Apoptotic and Autophagic Modulation by Nicotine
}

\section{Zahra Ahmadi' ${ }^{1}$, Milad Ashrafizadeh ${ }^{2}$, , Melika Forouzan' ${ }^{1}$}

${ }^{1}$ Department of basic science, Faculty of veterinary medicine, University of Shushtar, Khuzestan-Iran

${ }^{2}$ Department of basic science, Faculty of veterinary medicine, University of Tabriz, TabrizIran

*Corresponding Author E-mail: Dvm.milad73@yahoo.com

Received: 15 March 2019, Revised: 17 May 2019, Accepted: 25 May 2019

\begin{abstract}
Cigarette smoking is growing and nicotine is considered as the most adverse agent in the cigarette smoking. Nicotine is an alkaloid and forms $90 \%$ of alkaloids of cigarette. It is accountable for creating disorders and cancers such as lung cancer, heart disease and chronic lung disease. Nicotine can be considered as an autophagy and apoptosis modulator which can either directly or indirectly regulate apoptosis and autophagy. Apoptosis is the programmed cell death and oxidative stress in the most known inducer of apoptosis. However, physicochemical stresses, free radicals and removing of nutrients are other factors which induce apoptosis. Autophagy plays the role of recycling which mainly attacks damaged and aged cells and organelles. In this review, we investigate the effects of nicotine on these pathways.
\end{abstract}

Key words: Nicotine, Apoptosis, Autophagy, Cellular fate.

\section{Introduction}

During recent decades, much attention has been performed towards the toxicity of agents. Cigarette smoking has major effects on health and nicotine is considered as the most toxic agent in cigarette smoking. The nicotine usage is increasing, so that has become a habit. The mortality from cigarette smoking is high and lung cancer, heart disease and chronic lung diseases are three causes of death in smoking women. Many persons attempt for quitting smoking and finally due to the difficulty that is highest in first week, they give up and trigger smoking again. Nicotine is an alkaloid and is present in the leaves of tobacco where it acts as a botanical insecticide. A medium tobacco rod contains 10 to $14 \mathrm{mg}$ of nicotine and what is obvious is the detrimental effects of nicotine on the various parts of body. in this review, we investigate the apoptotic and autophagic pathways that are triggered by nicotine (Abdollahzadeh Soreshjani \& Ashrafizadeh, 2018a, 2018; Ahmadi \& Ashrafizadeh, 2018, 2019; Ahmadi, Mohammadinejad, \& Ashrafizadeh, 2019; Ashrafizadeh \& Ahmadi, 2019; Ashrafizadeh, Rafiei, \& Ahmadi, 2018; Hassanzadeh Davarania 2018; Hassanzadeh Davarani et al., 2018; Mohammadinejad, Dadashzadeh, et al., 2019; Rafiei, Ahmadi, \& Ashrafizadeh, 2018a, 2018b; Rafiei \& Ashrafizadeh, 2018; Sobhani, Roomiani, Ahmadi, \& Ashrafizadeh, 2019). 


\section{Materials and methods}

\section{Studied PatientsApoptosis}

Apoptosis or programmed cell death is an active process which is the main controlling system in various tissues. Apoptosis was first introduced as a cell death that happens in hepatocytes under physiological conditions (Kerr, 1965; Lockshin \& Zakeri, 2001). Interruption of membrane integrity, cell shrinking, fragmentation of chromatin condensation and inter-nucleosomal DNA as well as production of apoptotic bodies are morphological indications of apoptosis (Elmore, 2007; Jayakiran, 2015).

\section{Apoptosis mechanisms}

There are two important apoptotic pathways: Extrinsic or death receptor pathway and intrinsic or mitochondrial pathway. There is an additional pathway which acts via T-cell mediated cytotoxicity and perforin-granzyme-dependent killing of the cell.

The extrinsic signaling pathway depends on the death receptor transmembrane proteins which belong to the tumor necrosis factor (TNF) receptor gene superfamily. The bestknown ligands and corresponding death receptor are as following: FasL/FasR, TNF?/TNFR1, Apo3L/DR3, Apo2L/DR4 and Apo2L/DR5 (Ashkenazi \& Dixit, 1998; Chicheportiche et al., 1997; Locksley, Killeen, \& Lenardo, 2001; Peter \& Krammer, 1998; Rubio-Moscardo et al., 2005; Suliman, Lam, Datta, \& Srivastava, 2001). Binding of ligand to the receptor results in recruiting adaptor protein FADD (Fas associated via death domain). FADD is associated to the procaspase-8 through dimerization of death effector domain and death-inducing signaling complex (DISC) is formed. After autocatalytic activation of procaspase-8, caspase-8 is activated which demonstrates the execution phase of apoptosis. Intrinsic signaling pathway is triggered by the permeability of mitochondrial membrane. The stimulations which initiate this pathway, are divided into two categories: negative and positive stimulations. In negative fashion, loss of certain growth factors, hormones and cytokines (factors suppressing death programs) results in apoptosis, whereas in positive fashion, radiation, toxins, hypoxia, hyperthermia, viral infections and free radicals involve (Birnbaum, Clem, \& Miller, 1994). Bcl-2 superfamily proteins which regulate the mitochondrial integrity, are divided into two categories: Anti- and pro-apoptotic proteins. Pro-apoptotic family includes BAX, BID, BAK1, BAD, PMAIP1/Noxa and BBC3/Puma and anti-apoptotic family includes Bcl-2, Bcl2L1/Bcl-xl, MCL-1 and Bcl2A1 (Black, Kronheim, \& Sleath, 1989). The stimulations (negative and positive stimulations) result in alterations in inner membrane of mitochondria, leading to the opening of mitochondrial permeability transition (MPT) pore. Then, pro-apoptotic proteins activate caspase-dependent mitochondrial pathway. Cytochrome-C binds and activates Apaf-1 and procaspase-9, resulting in formation of apoptosome (Chinnaiyan, 1999; Hill, Adrain, Duriez, Creagh, \& Martin, 2004). Recruiment and activation of CASP9 is performed by apoptosome, leading to the activation of CASP3, CASP6 and CASP7 and finally, results in apoptosis.

Endoplasmic reticulum (Fatemeh Hassanzadeh Davarania) pressures trigger the third pathway of apoptosis (Ashrafizadeh et al.,). The unfold protein response (UPR) accounts for dealing with ER tension and unresolved pressure leads to the beginning of apoptosis. CASP12 and CASP4 are respectively responsible for mediation of ER-induced apoptosis in rodents and human. Besides, Bcl-2 family proteins control the apoptosis via direct or indirect $\left(\mathrm{ER} \mathrm{Ca}^{2+}\right.$ signaling) pathways. 


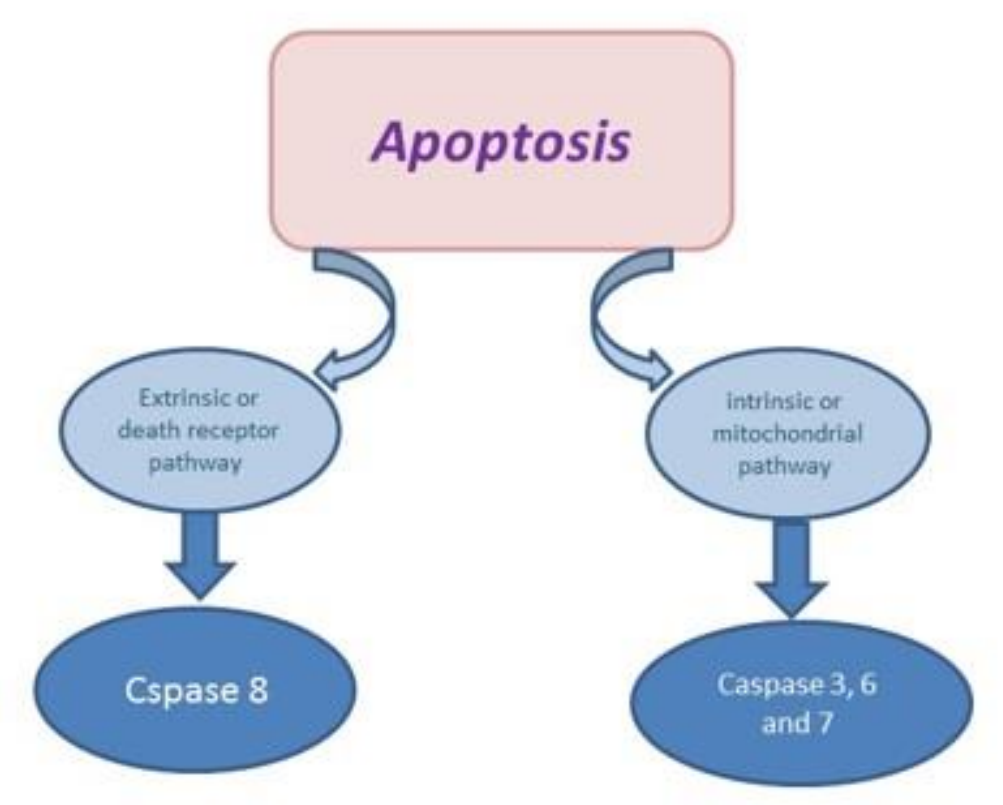

Figure 1. Schematic presentation of intrinsic and extrinsic pathways of apoptosis

\section{How the apoptosis is stimulated?}

It has been shown that apoptosis can occur in all metazoan and protozan cells and it is considered as a potential survival mechanism, because this mechanism eliminates the defective or damaged cells as well as cells which are known as a menace for the completeness of the whole organism. The stimulations in the normal or pathophysiological conditions can induce the apoptosis(Toivola, Strnad, Habtezion, \& Omary, 2010; Wyllie, 2010). There are different kinds of stimuli which can stimulate apoptosis (Portt, Norman, Clapp, Greenwood, \& Greenwood, 2011). Physicochemical stresses including chemotherapeutic compunds (doxorubicin), changes in temperature and osmolarity, DNA damaging materials, compounds producing free radicals, elimination of nutrients, oxygen or growth factors, pro-inflammatory cytokines an-d normal physiological processes such as ageing and development are examples of these stimuli. Also, ischemia and accumulation of misfolded ER proteins are pathological stimuli. There are evidences about the association between various apoptosis pathways, so that, for instance, extrinsic pathway can trigger the mitochondrial pathway. For example, it has been reported that BBC3/Puma and PMAIP1/Noxa can begin the ER-mediated apoptosis with the contribution of mitochondrial apoptosis pathway (Fink \& Cookson, 2005).

\section{Autophagy}

In recent years, autophagy has attracted a remarkable attention due to its mediation between death and life (Mohammadinejad, Ahmadi, Tavakol, \& Ashrafizadeh, 2019). Autophagy is a catabolic process which has been conserved in eukaryotic organisms. It functions as a cytoprotective mechanism which regulates metabolic functions inside the cells, ageing, cancer, neurodegenerative disorders and lysosomal disorders, demonstrating the major role of autophagy in regulating homeostatis. Autophagy by breaking proteins and 
peptides and providing a pool of aminoacids, contributes the cells to survive in stress conditions such as nutrient starvation and ATP deficiency. Autophagy adjusts intracellular conditions via cytoplasmic turnover of proteins and organelles, so that after deletion of special autophagic proteins (Atg5 and Atg7), the accumulation of ubiquitted proteins happens, showing the important role of autophagy in modulating the protein turnover of cells (Komatsu et al., 2005). Also, mutant yeasts with defective autophagy demonstrated devastated spore formation, showing the role of autophagy in maintaining cell growth and development (Tsukada \& Ohsumi, 1993).

Mechanistic target of rapamycin kinase (mTOR), 2 autophagy-promoting kinases, 5/-AMP activated protein kinase (AMPK) and class II phosphatidylinositol-3-kinase (PtdIns3k) are major signaling pathways which determine the stimulation and magnitude of autophagy. Generally, in stress conditions, autophagy activates AMPK which phosphorylates the members of ULK1 kinase and PtdIns3k complexes and also inhibition of mTOR occurs. Bcl2 , an inhibitor of BECN1, plays an important role in the association of autophagy and apoptosis. The under short-term stress conditions MAPK8/JNK1 phosphorylation leads to the separation of Bcl-2 from BECN1 and activation of PtdIns3k and finally advancement of autophagy, but long-term phosphorylation might result in inactivation of $\mathrm{Bcl}-2$ and induction of apoptosis (Wei, Pattingre, Sinha, Bassik, \& Levine, 2008).

\section{Nicotine and apoptosis}

There are different studies about the effects of nicotine on the induction of apoptosis. Kang et al studied the nicotine-induced apoptosis in human gingivial fibroblasts (HGFs) (Kang et al., 2011). Their results showed that nicotine increases the level of BAX (pro-apoptotic protein) and Bcl-2 (anti-apoptotic protein) in HGFs. Also, nicotine results in releasing cytochrome c from mitochondria to cytosol and activates caspase -3 and -9 . Besides, poly ADP polymerase (PARP) degradation occurs as a result of nicotine exposure. The transmission of oxidative stimuli in cytoplasm and signals of membrane surface receptors to the nucleus might be occurred to regulate the cell division and survival. Oxidative stress leads to the activation of many protein kinases and transcription regulatory factors(Kalariya, Wills, Ramana, Srivastava, \& van Kuijk, 2009; Y.S. Lee et al., 2009). Nicotine increases the synthesis of ROS and negatively affects the survival and differentiation of cell. Also, activation of MAPKs such as ERK, JNK and P38 MAPK is associated with the transmission of apoptosis stimuli caspases (Chuang, Wang, \& Yang, 2000; Platanias, 2003). It has been shown that nicotine activates ERK, JNK and P38 MAPK to induce apoptosis.

Three other studies also examined the apoptotic effect of nicotine on cell (H.J. Lee et al., 2008; Y. LIU, 2004; Tian, $\mathrm{Zhu}, \mathrm{Li}, \mathrm{Ma}, \& \mathrm{Wu}, 2009$ ). The first study demonstrated that nicotine induces mitochondrial apoptotic pathway to block the viability, proliferation and differentiation of human umbilical endothelial cells. Second study examined the cytotoxic effects of nicotine on alveolar epithelial cells and showed that nicotine activates betacatenin/TCF signaling via inhibiting GSK3 beta. Also, third study demonstrated the involvement of PI3K, MAPK and NF-kappa B pathways in cytotoxicity effects of nicotine on immortalized and malignant human oral keratinocytes. Kim et al studied the nicotineinduced apoptosis in leydig cells (Kim et al., 2005). They used various methods to show that nicotine stimulates apoptosis in leydig cells. Their results demonstrated that nicotine affects leyding cells in a dose- and time-dependent manner and induces cell shrinkage, 
cytoplasmic condensation and irregularity in shape in these cells. Also, apoptotic bodies (as markers of apoptosis), cytoplasmic blebbing, nuclear shrinkage and chromatin condensation have been observed at the result of exposing to nicotine. Zanetti et al., also showed that nicotine exerts its cytotoxicity via oxidative stress (Zanetti et al., 2014). Recently, Marinucci et al., discovered a new mechanism that nicotine uses to stimulate apoptosis (Marinucci et al., 2018). They used human primary osteoblasts and their results showed that nicotine remarkably increases the intracellular accumulation of $\mathrm{H} 2 \mathrm{O} 2$ via inhibiting Glo1 which induces MG-H1 accumulation/release and MG-H1 starts H2O2 overproduction by RAGE and an apoptotic mitochondrial pathway by stimulating TG2 downregulation-dependent NF-kB desensitization. In a study, Amini and colleagues evaluated the effect of nicotine on the PC12 cells (Amini, Zhaleh, Tahvilian, \& Farnia, 2019). The results of this study demonstrated that nicotine consumption is associated with reduction in cell viability, cell proliferation, an increase in cell death and elevated concentrations of intracellular and mitochondrial calcium. In another study, Yang and coworkers investigated the impact of nicotine on the proliferation and migration of human primary pterygium cell (Yang et al., 2019). It was demonstrated that exposing to the nicotine for a long time suppresses cell proliferation and migration. Besides, nicotine has this ability to negatively affect human fetal ovaries (Cheng et al., 2018). In this study, it was found that nicotine exposure remarkably enhances the generation of ROS and DNA damage, consequently resulting in apoptosis and decreased viability of human fetal ovaries. Another study conducted by Mohammadghasemi and Jahromi, confirmed that exposing mice to the nicotine $(0.1 \mathrm{mg} / 100 \mathrm{mg})$ significantly stimulates apoptosis and testicular damage and melatonin can be considered as a potential candidate in diminishing apoptosis (Mohammadghasemi \& Jahromi, 2018).

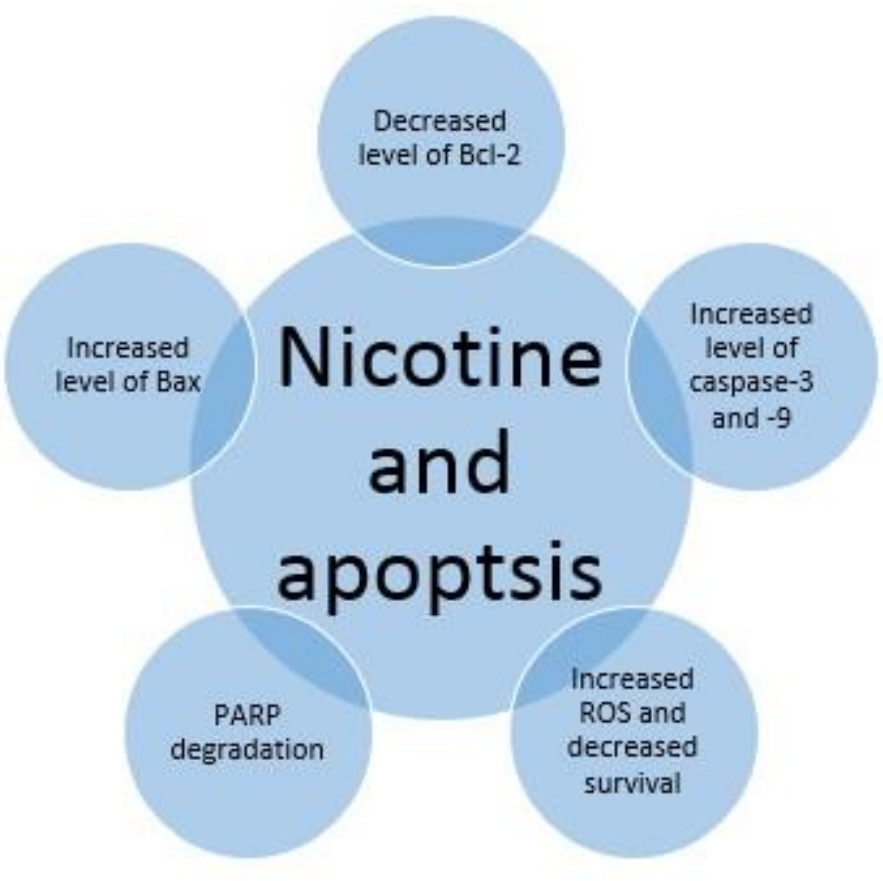

Figure 2. The association between nicotine and apoptosis 


\section{Nicotine and autophagy}

As we mentioned, autophagy and apoptosis are in relationship with each other and both of them play a significant role in cell survival and death. Autophagy has attracted much attention during past decades. So far, it has been shown that apoptosis is the major mechanism that nicotine uses and there is a little information about the autophagy pathway. Recently, it was shown that autophagy is the major mechanism which nicotine applies to diminish the serum testosterone and researchers thought that apoptosis is the cause of this diminution. It was demonstrated that nicotine starts autophagy via the activity of CHRNA7. These results about the role of nicotine in stimulating autophagy is in agreement with the study of Xiao et al., (Xiao, Shang, Zhai, Zhang, \& Zhang, 2018). They traced Beclin-1 and LC3 antibodies to investigate autophagy. Their data showed that nicotine at the dose of $0.2 \mathrm{mg} / \mathrm{kg}$ can trigger autophagy and can be considered as a beneficial agent for inhibiting the anxiety and depressive-like behavior. Also, an interesting research showed that even apoptosis caused by nicotine is the result of interruption and disorder in autophagy (Bodas, Van Westphal, Carpenter-Thompson, Mohanty, \& Vij, 2016). In a study, the stimulatory effect of nicotine on the atherosclerosis was examined (Z. Wang, Liu, Zhu, Wang, \& Wang, 2019). The results of this study revealed that nicotine predisposes to atherosclerosis by induction of autophagy through enhancing LC3II to LC3I ratio. Wang and colleagues indicated that nicotine effectively stimulates autophagy, leading to the adverse effects on mouse early folliculogenesis (Y.F. Wang et al., 2018). Xia and coworkers assessed the impact of nicotine on the chronic stress-induced anxiety and depressive-like behavior (Xiao et al., 2018). It was found that nicotine activates autophagy mechanism through elevating the expression of Beclin-1 and LC3II, resulting in attenuation of neurological disorder and hippocampal neuropathy. These findings were in line with the research of Liu and colleagues that demonstrated the adverse effects of nicotine on leydig cells via stimulation of autophagy (S. Liu, Sun, \& Li, 2018).

\section{Conclusion}

Nicotine is the most harmful compound in the cigarette smoking and apoptotic as well as autophagic fates that are affected by nicotine, are important in terms of directing future researches for understanding the exact mechanism of triggering autophagy and apoptosis by nicotine. In summary, nicotine induces apoptosis in cells and can be used in cancer therapy because of its cytotoxicity on cancer cell lines. Also, it begins autophagy and maybe in the future can be used as an autophagy inducer to resolve the problems that are associated with autophagy impairment.

\section{Conflict of interest}

The authors declare no conflict of interest.

\section{References}

Abdollahzadeh Soreshjani, S, Ashrafizadeh, M. (2018a). Effects of exercise on testosterone level, heat shock protein, and fertility potential. Reviews Clin. Med., 5(4):141-145. 
Abdollahzadeh Soreshjani, S, Ashrafizadeh, M. (2018b). The Effects of the Exercise on the Testosterone Level, Heat Shock Proteins and Fertility Potential. Reviews Clin. Med., 5(4):1215.

Ahmadi, Z, Ashrafizadeh, M. (2018). Downregulation of osteocalcin gene in chickens treated with Lead Acetate II. Int. Biolog. Biomed. J., 4(4):0-0.

Ahmadi, Z, \& Ashrafizadeh, M. (2019). Down Regulation of Osteocalcin Gene in Chickens Treated with Cadmium. Iranian J. Toxicol., 13(1):1-4.

Ahmadi, Z, Mohammadinejad, R, Ashrafizadeh, M. (2019). Drug delivery systems for resveratrol, a non-flavonoid polyphenol: Emerging evidence in last decades. J. Drug Delivery Sci. Technol.

Amini, K, Zhaleh, H, Tahvilian, R, Farnia, V. (2019). Low concentration of morphine protects against cell death, oxidative stress and calcium accumulation by nicotine in PC12 cells. Bratisl. Lek. listy, 120(4):256-262.

Ashkenazi, A, Dixit, VM. (1998). Death receptors: signaling and modulation. science, 281(5381):1305-1308.

Ashrafizadeh, M, Ahmadi, Z. (2019). The effects of astaxanthin treatment on the sperm quality of mice treated with nicotine. Reviews in Clinical Medicine, 6(1):156-158.

Ashrafizadeh, M, Mohammadinejad, R, Tavakol, S, Ahmadi, Z, Roomiani, S, Katebi, M. Autophagy, anoikis, ferroptosis, necroptosis, and endoplasmic reticulum stress: Potential applications in melanoma therapy. Journal of Cellular Physiology.

Ashrafizadeh, M, Rafiei, H, Ahmadi, Z. (2018). Histological changes in the liver and biochemical parameters of chickens treated with lead acetate II. Iranian J. Toxicol., 12(6):15 .

Birnbaum, M, Clem, R, Miller, L. (1994). An apoptosis-inhibiting gene from a nuclear polyhedrosis virus encoding a polypeptide with Cys/His sequence motifs. J. virol., 68(4):2521-2528.

Black, RA, Kronheim, SR, Sleath, PR. (1989). Activation of interleukin-1 $\beta$ by a co-induced protease. FEBS lett., 247(2):386-390.

Bodas, M, Van Westphal, C, Carpenter-Thompson, R, Mohanty, DK, Vij, N. (2016). Nicotine exposure induces bronchial epithelial cell apoptosis and senescence via ROS mediated autophagy-impairment. Free Radic. Biol. Med., 97:441-453.

Cheng, SF, Qin, XS, Han, ZL, Sun, XF, Feng, YN, Yang, F, . . . De Felici, M. (2018). Nicotine exposure impairs germ cell development in human fetal ovaries cultured in vitro. Aging (Albany NY), 10(7):1556.

Chicheportiche, Y, Bourdon, PR, Xu, H, Hsu, YM, Scott, H, Hession, C, Garcia, I, Browning, JL. (1997). TWEAK, a new secreted ligand in the tumor necrosis factor family that weakly induces apoptosis. J. Biol. Chem., 272(51):32401-32410. 
Chinnaiyan, AM. (1999). The apoptosome: heart and soul of the cell death machine. Neoplasia, 1(1):5-15.

Chuang, SM, Wang, IC, Yang, JL. (2000). Roles of JNK, p38 and ERK mitogen-activated protein kinases in the growth inhibition and apoptosis induced by cadmium. Carcinogenesis, 21(7):1423-1432.

Elmore, S. (2007). Apoptosis: a review of programmed cell death. Toxicol. Pathol., 35(4):495-516.

Fatemeh Hassanzadeh Davarania, M. A., Roohollah Saberi Risehc, Elham Ghasemipour Afshard , Hamid Mohammadie, Seyed Hamid Razavif, Ali Mandegaryg, Reza Mohammadinejadh*. (2018). Antifungal nanoparticles reduce aflatoxin contamination in pistachio. Pistachio and Health journal.

Fink, SL, Cookson, BT. (2005). Apoptosis, pyroptosis, and necrosis: mechanistic description of dead and dying eukaryotic cells. Infect. Immune., 73(4):1907-1916.

Hassanzadeh Davarani, F, Ashrafizadeh, M, Saberi Riseh, R, Ghasemipour Afshar, E, Mohammadi, H, Razavi, SH, Mandegary A, Mohammadinejad, R. (2018). Antifungal nanoparticles reduce aflatoxin contamination in pistachio. Pistachio Health J., 1(2):26-33. doi:10.22123/phj.2018.130469.1007

Hill, MM, Adrain, C, Duriez, PJ, Creagh, EM, Martin, SJ. (2004). Analysis of the composition, assembly kinetics and activity of native Apaf-1 apoptosomes. EMBO J., 23(10):2134-2145.

Jayakiran, M. (2015). Apoptosis-biochemistry: a mini review. J. Clin. Exp. Pathol., 5(1):1-4.

Kalariya, NM, Wills, NK, Ramana, KV, Srivastava, SK, van Kuijk, FJ. (2009). Cadmium-induced apoptotic death of human retinal pigment epithelial cells is mediated by MAPK pathway. Exp. Eye Res., 89(4):494-502.

Kang, SW, Park, HJ, Ban, JY, Chung, JH, Chun, GS, Cho, JO. (2011). Effects of nicotine on apoptosis in human gingival fibroblasts. Arch. Oral Boil., 56(10):1091-1097.

Kerr, JFR. (1965). A histochemical study of hypertrophy and ischaemic injury of rat liver with special reference to changes in lysosomes. J. pathol. Bacterial., 90(2):419-435.

Kim, KH, Joo, KJ, Park, HJ, Kwon, CH, Jang, MH, Kim, CJ. (2005). Nicotine induces apoptosis in TM3 mouse Leydig cells. Fertil. Steril., 83(4):1093-1099.

Komatsu, M, Waguri, S, Ueno, T, Iwata, J, Murata, S, Tanida, I, . . Uchiyama, Y. (2005). Impairment of starvation-induced and constitutive autophagy in Atg7-deficient mice. J. Cell. Biol., 169(3):425-434.

Lee, HJ, Lee, J, Min, SK, Guo, HY, Lee, SK, Kim, HR, ... Lee, SK. (2008). Differential induction of heme oxygenase-1 against nicotine-induced cytotoxicity via the PI3K, MAPK, and NF-kappa B pathways in immortalized and malignant human oral keratinocytes. J. Oral Pathol. Med., 37(5):278-286. 
Lee, YS, Yoon, HJ, Oh, JH, Park, HJ, Lee, EH, Song, CW, Yoon, S. (2009). 1,3-Dinitrobenzene induces apoptosis in TM4 mouse Sertoli cells: Involvement of the c-Jun N-terminal kinase (JNK) MAPK pathway. Toxicol. Lett., 189(2):145-151.

Liu, S, Sun, Y, Li, Z. (2018). Resveratrol protects Leydig cells from nicotine-induced oxidative damage through enhanced autophagy. Clin. Exp. Pharmacol. Physiol., 45(6):573580.

LIU, Y. (2004). Damaging effect of cigarette smoke extract on primary cultured human umbilical vein endothelial cells and its mechanism. Biomed. Environ. Sci., 17:121-134.

Lockshin, RA, Zakeri, Z. (2001). Programmed cell death and apoptosis: origins of the theory. Nat. Rev. Mol. Cell Boil., 2(7):545.

Locksley, RM, Killeen, N, Lenardo, MJ. (2001). The TNF and TNF receptor superfamilies: integrating mammalian biology. Cell, 104(4):487-501.

Marinucci, L, Balloni, S, Fettucciari, K, Bodo, M, Talesa, VN, Antognelli, C. (2018). Nicotine induces apoptosis in human osteoblasts via a novel mechanism driven by $\mathrm{H} 2 \mathrm{O} 2$ and entailing Glyoxalase 1-dependent MG-H1 accumulation leading to TG2-mediated NF-kB desensitization: Implication for smokers-related osteoporosis. Free Radical Biol. Med., 117:6-17.

Mohammadghasemi, F, Jahromi, SK. (2018). Melatonin ameliorates testicular damages induced by nicotine in mice. Iranian J. Basic Med. Sci., 21(6):639.

Mohammadinejad, R, Ahmadi, Z, Tavakol, S, Ashrafizadeh, M. (2019). Berberine as a potential autophagy modulator. Journal of Cellular Physiology.

Mohammadinejad, R, Dadashzadeh, A, Moghassemi, S, Ashrafizadeh, M, Dehshahri, A, Pardakhty, A., ... Mandegary, A. (2019). Shedding light on gene therapy: carbon dots for the minimally invasive image-guided delivery of plasmids and noncoding RNAs. Journal of advanced research.

Peter, ME, Krammer, PH. (1998). Mechanisms of CD95 (AP0-1/Fas)-mediated apoptosis. Curr. Opin. Immunol., 10(5):545-551.

Platanias, LC. (2003). The p38 mitogen-activated protein kinase pathway and its role in interferon signaling. Pharmacol. Ther., 98(2), 129-142.

Portt, L, Norman, G, Clapp, C, Greenwood, M, Greenwood, MT. (2011). Anti-apoptosis and cell survival: a review. Biochim. Biophys. Acta (BBA)-Mol. Cell Res., 1813(1):238-259.

Rafiei, H, Ahmadi, Z, Ashrafizadeh, M. (2018a). Effects of orally administered lead acetate II on rat femur histology, mineralization properties and expression of osteocalcin gene. Int. Biolog. Biomed. J., 4(3):149-155.

Rafiei, H., Ahmadi, Z., \& Ashrafizadeh, M. (2018b). Effects of Orally Administered Lead acetate II on Rat Femur Histology, Mineralization Properties and Expression of Osteocalcin Gene. Int. Biolog. Biomed. J., 4(3):0-0. 
Rafiei, H, Ashrafizadeh, M. (2018). Expression of collagen type II and osteocalcin genes in mesenchymal stem cells from rats treated with lead acetate II. Iranian J. Toxicol., 12(5):3540.

Rubio-Moscardo, F, Blesa, D, Mestre, C, Siebert, R, Balasas, T, Benito, A., ... Schilhabel, M. (2005). Characterization of 8p21. 3 chromosomal deletions in B-cell lymphoma: TRAIL-R1 and TRAIL-R2 as candidate dosage-dependent tumor suppressor genes. Blood, 106(9), 3214-3222.

Sobhani, B, Roomiani, S, Ahmadi, Z, Ashrafizadeh, M. (2019). Histopathological Analysis of Testis: Effects of Astaxanthin Treatment against Nicotine Toxicity. Iranian J. Toxicol, 13(1):41-44.

Suliman, A, Lam, A, Datta, R, Srivastava, RK. (2001). Intracellular mechanisms of TRAIL: apoptosis through mitochondrial-dependent and-independent pathways. Oncogene, 20(17):2122.

Tian, D, Zhu, M, Li, J, Ma, Y, Wu, R. (2009). Cigarette smoke extract induces activation of $\beta$ catenin/TCF signaling through inhibiting GSK3 $\beta$ in human alveolar epithelial cell line. Toxicol. Lett., 187(1):58-62.

Toivola, D, Strnad, P, Habtezion, A, Omary, M. (2010). Intermediate filaments take the heat as stress proteins. Trend. cell boil., 20(2):79-91.

Tsukada, M, Ohsumi, Y. (1993). Isolation and characterization of autophagy-defective mutants of Saccharomyces cerevisiae. FEBS lett., 333(1-2):169-174.

Wang, YF, Sun, XF, Han, ZL, Li, L, Ge, W, Zhao, Y, De Felici, M, Shen, W, Cheng, SF. (2018). Protective effects of melatonin against nicotine-induced disorder of mouse early folliculogenesis. Aging (Albany NY), 10(3):463.

Wang, Z, Liu, B, Zhu, J, Wang, D, Wang, Y. (2019). Nicotine-mediated autophagy of vascular smooth muscle cell accelerates atherosclerosis via nAChRs/ROS/NF- $\mathrm{kB}$ signaling pathway. Atherosclerosis, 284:1-10.

Wei, Y, Pattingre, S, Sinha, S, Bassik, M, Levine, B. (2008). JNK1-mediated phosphorylation of Bcl-2 regulates starvation-induced autophagy. Mol. Cell, 30(6):678-688.

Wyllie, AH. (2010). "Where, O death, is thy sting?" A brief review of apoptosis biology. Mol. Neurobiol., 42(1):4-9.

Xiao, X, Shang, X, Zhai, B, Zhang, H, Zhang, T. (2018). Nicotine alleviates chronic stressinduced anxiety and depressive-like behavior and hippocampal neuropathology via regulating autophagy signaling. Neurochem. Int., 114:58-70.

Yang, Q, Jhanji, V, Tan, SQ, Chan, KP, Cao, D, Chu, WK, Zhang, M, Pang, CP, Ng, TK. (2019). Continuous exposure of nicotine and cotinine retards human primary pterygium cell proliferation and migration. J. Cell. Biochem., 120(3):4203-4213. 
Zanetti, F, Giacomello, M, Donati, Y, Carnesecchi, S, Frieden, M, Barazzone-Argiroffo, C. (2014). Nicotine mediates oxidative stress and apoptosis through cross talk between NOX1 and Bcl-2 in lung epithelial cells. Free Radic. Biol. Med., 76:173-184.

How to cite this article: Zahra Ahmadi, Milad Ashrafizadeh, Melika Forouzan. Apoptotic and Autophagic Modulation by Nicotine. International Journal of Advanced Biological and Biomedical Research, 2019, 7(3), 263-273. Link:

http://www.ijabbr.com/article 35336.html 